\title{
An Investigation into the Effects of Stevia on food intake, satiety and blood glucose levels in adults: a small-scale study
}

\section{Abstract}

Non-nutritive sweeteners (NNS) are suggested to reduce sugar and energy content of diet, but there is growing evidence that they can exacerbate obesity and diabetes through increasing appetite and energy intake. Stevia (stevioside extract), a natural sweetener, is being increasingly consumed, yet limited studies have looked at their effects on satiety and energy intake. The aim of this study is to investigate the effects of preloads of stevia on food intake, satiety and postprandial blood glucose levels when compared to water and sugar. Thirty participants (10 males/20 females; $26 \pm 10.5$ years; BMI: $23.44 \pm 3.42 \mathrm{Kg} / \mathrm{m}^{2}$ ) took part in a three-arm single-blinded crossover trial. On separate test days, they received three different preloads $(300 \mathrm{ml})$ containing water, sugar $(60 \mathrm{~g})$ and stevia $(1 \mathrm{~g})$ followed by an ad-libitum pizza lunch after 30 minutes. Breakfast was standardized. Blood glucose samples were collected before preload and lunch, and then at 30-minute intervals until 120 min post lunch. Volunteers recorded their feelings of satiety and hunger on visual analogue scales (VAS) before preload and after meal intake. A one-day diet diary was collected for each test day. Data was analysed using repeated measures ANOVA (SPSS Inc., Chicago, IL, USA). Despite the difference in energy content between preloads, there were no significant differences in energy intake at lunch between the three interventions $(\mathrm{F}(2,56)=0.25, p=0.78)$. Furthermore, participants did not compensate by consuming more energy during the day after the stevia preload (1660 \pm 584 Kcal) compared to sugar preload $(1770 \pm 763 \mathrm{Kcal}, p=0.82)$. There were no significant differences in VAS scores between stevia and sugar preloads, but participants scored significantly higher rates of hunger (before and after lunch) and desire to eat (before lunch) following water preload $(p<0.05)$. No significant differences between water, sugar and stevia were noted for postprandial glucose levels $(120$ min post lunch) when adjusted from baseline $(\mathrm{F}(2,58)=2.56, p=0.09)$. Area under the curve (AUC) for glucose did not differ between water and stevia $(p=0.2)$. Results are in line with several clinical trials showing that the consumption of sweeteners does not lead to an increase in hunger and energy intake, and could therefore present a useful strategy to assist with weight loss. Further studies looking at long-term effects of stevia on weight regulation are needed to support these findings.

\section{Conflict of Interest}

There is no conflict of interest. 